\title{
Impact of Ventricular Assist Device Implantation on Clinical and Economic Outcomes of Heart Transplantation in the United States from 2003-2007
}

\author{
Julie W. Doberne, Brian Diggs, Frederick Tibayan, Matthew Slater, \\ Christopher V. Chien, Jill Gelow, James Mudd, Howard K. Song \\ Divisions of Cardiothoracic Surgery and Cardiovascular Medicine, Oregon Health \& Science University, Portland, USA \\ Email: songh@ohsu.edu
}

Received July 25, 2013; revised August 22, 2013; accepted August 31, 2013

Copyright (C) 2013 Julie W. Doberne et al. This is an open access article distributed under the Creative Commons Attribution License, which permits unrestricted use, distribution, and reproduction in any medium, provided the original work is properly cited.

\begin{abstract}
The use of ventricular assist devices (VADs) as a bridge to transplantation (BTT) has evolved considerably as clinical experience has grown and newer generation devices have become available. This study investigated the impact of VAD implantation on the clinical and economic outcomes of patients undergoing heart transplantation in the United States (US) from 2003-2007. A retrospective study was carried out using the Nationwide Inpatient Sample, to track the characteristics and outcomes of an estimated 7859 patients undergoing heart transplantation in the United States from 2003-2007. Patients were divided based on whether they were bridged to transplant with a VAD and whether they were outpatient (VAD Outpt) or inpatient (VAD Inpt). Multivariate analysis was performed to identify risk factors for inhospital mortality following heart transplantation. Hospital costs were also measured. A VAD was used as a BTT in $20.8 \%$ of patients undergoing heart transplantation during the study period. Early in the series the frequency of VAD Outpts and Inpts was similar but in later years patients were more commonly bridged as outpatients. Hospital mortality for the entire population was $6.2 \%$. Multivariate analysis identified several risk factors for mortality, including advanced age, history of congenital heart disease, and VAD Inpt status. VAD Inpt status was the strongest predictor of mortality. The hospital cost of patients bridged as inpatients was more than double the cost of outpatients. Median hospital cost declined by $11 \%$ over the study period, from $\$ 115,868$ to $\$ 103,578$. Much of this cost reduction was related to the shift in VAD status from Inpt to Outpt. Heart transplant recipients are increasingly bridged to transplantation with VADs as outpatients. Those bridged as inpatients have substantially higher hospital mortality and cost. These findings have important clinical implications and should inform policy development, resource utilization and donor allocation.
\end{abstract}

Keywords: Ventricular Assist Device; Heart Transplantation; Cost Effectiveness

\section{Introduction}

Ongoing limitations in donor heart supply and improvements in ventricular assist device (VAD) technology have led to increased utilization of VADs as a bridge to transplantation [1-5]. Decreasing mortality and cost of VAD usage in the post-REMATCH era suggest improvements in care and resource utilization for patients requiring mechanical circulatory support [6]. The impact of prior VAD implantation on heart transplant outcomes has been assessed extensively but uncertainty persists on how the BTT pathway affects transplant outcomes and how this pathway can be optimized for greatest net benefit to the heart transplant population and to the healthcare system [7-11]. Currently, there is considerable variation among heart transplant centers in the utilization of VADs as BTT and the clinical circumstances under which patients are selected for this therapy.

This study was undertaken to investigate the impact of ventricular assist device implantation on the clinical and economic outcomes of patients undergoing heart transplantation in the United States. A retrospective study was carried out utilizing the Nationwide Inpatient Sample (NIS), a project of the Agency for Healthcare Research and Quality [12]. This database is the largest all-payer inpatient database publicly available in the United States and allows analysis of national trends in health care 
utilization, outcomes, and charges. Given the current economic challenges faced by the health care system, studies of treatments and treatment pathways that measure quality as well as cost will be important to assess the efficiency of health care delivery.

\section{Materials and Methods}

Data were selected from the NIS combining years 20032007. The NIS is a stratified randomized $20 \%$ sample of patient discharge abstracts from non-federal hospitals. In combining the multiple years of NIS data, the revised statistical weights for comparisons across time were used.

Cases were included in the analysis if they had a recorded ICD-9 procedure code of VAD implantation, 37.62 ("Insertion of non-implantable heart assist system"), 37.65 ("Implant of external heart assist system"), or 37.66 ("Insertion of implantable heart assist system"); explant codes 37.64 ("Removal of external heart assist system(s) or device(s)") or 97.44 ("explantation [removal] of percutaneous external heart assist device"); and lastly, heart transplantation codes 37.5 or 37.51 ("Heart transplantation").

Patient comorbidities were defined based on diagnosis codes present in the discharge abstract.

Charge data were adjusted for inflation using the Consumer Price Index published by the US Department of Labor, Bureau of Labor Statistics. The specific series used were US city average all items (CUUS0000SA0) and US city average medical care services (CUUS0000SAM2). The medical care services series includes professional services, hospital and related services, and health insurance. Charge data were normalized to 2007 dollars.

This study was approved by the Institutional Review Board at Oregon Health \& Science University. Individual consent was waived.

\section{Results}

Adult patients (age 18 years or greater) in the NIS database undergoing heart transplantation from 2003-2007 were identified (Table 1). The estimated number of patients captured by the NIS database over this period was 7859. The number of adult heart transplant recipients in the United States tracked by the Organ Procurement and Transplantation Network over the same period was 9066 [13]. The NIS database therefore provided an $86.7 \%$

Table 1. Number of heart transplant procedures in the United States from 2003-2007 identified in the NIS database.

\begin{tabular}{ccccccc}
\hline Year & 2003 & 2004 & 2005 & 2006 & 2007 & Total \\
\hline Number of Transplants & 1845 & 1191 & 1719 & 1686 & 1418 & 7859 \\
\hline
\end{tabular}

sample for patients undergoing heart transplantation in the United States, sufficient to perform an analysis of clinical and financial outcomes.

We evaluated the impact of VAD utilization on heart transplant outcomes. The frequency of VAD utilization as a bridge to transplantation was tracked over the study period (Figure 1). The overall frequency of VAD utilization as a bridge to heart transplantation (VAD Total) increased over the five year period from $14.0 \%$ to $21.2 \%$. The frequency of patients bridged to transplant solely as inpatients (VAD Inpt) was stable over the study period. The NIS database does not contain data fields that allow discrimination between VAD type or indication for left (LVAD), right (RVAD), or biventricular support (BiVAD).

The demographic summary of adults undergoing heart transplantation in the NIS database is listed in Table 2. The demographic data for the NIS heart transplant population is similar to previously published reports describing the United States heart transplant and other cardiac surgery patient population $[14,15]$. The majority of heart transplant recipients in the NIS database for this time period were male $(75 \%)$ and had a primary diagnosis of ischemic heart disease or cardiomyopathy. The NIS database also provides information on the hospital setting and health insurance status of recipients.

Hospital mortality following heart transplantation varied considerably based on specific VAD status, particularly between inpatients and outpatients. The hospital mortality for all patients over the study period was $6.2 \%$ (Table 3). This overall mortality rate is consistent with previously published studies reporting early mortality following heart transplantation in the United States and abroad over a similar era [7,16,17]. Patients not supported with a VAD or supported with a VAD as an outpatient had similarly low hospital mortality. Patients supported with a VAD as a BTT as inpatients had a strikingly higher mortality of $25.6 \%$.

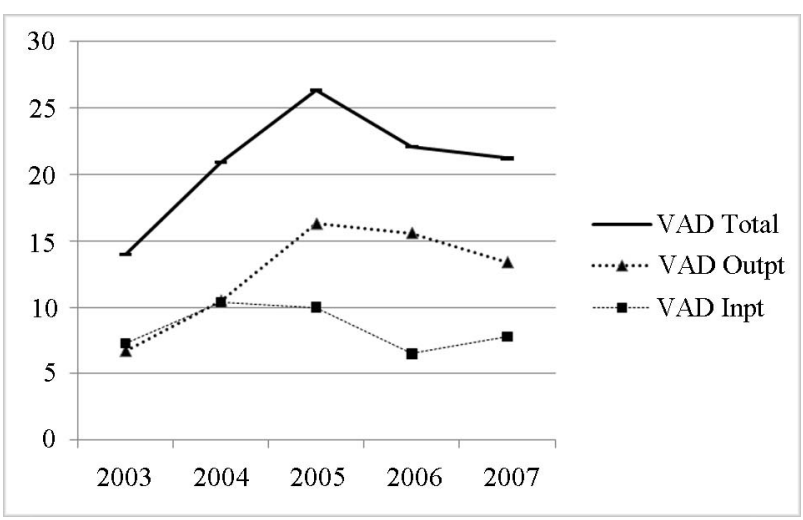

Figure 1. Frequency of VAD utilization as bridge to transplantation in the United States from 2003-2007. 
Table 2. Demographic information of adult patients undergoing heart transplant in the NIS database from 2003-2007.

\begin{tabular}{cccc}
\hline Characteristic & No BTT (n = 6227) & VAD Outpt (n = 983) & VAD Inpt (n = 649) \\
\hline Age, mean (SD), y & $51(28)$ & $50(27)$ & $47(30)$ \\
Male (\%) & 73.1 & 82.4 & 80.7 \\
Underlying diagnosis (\%) & & & 4.7 \\
Congenital & 3.6 & 4.6 & 46.5 \\
Ischemia & 44.9 & 47.8 & 39.8 \\
Cardiomyopathy & 47.5 & 6.1 & 9.0 \\
Other & 4.1 & & 95.5 \\
Hospital type (\%) & & 94.3 & 4.5 \\
Urban, teaching & 93.9 & 5.7 & \\
Urban, nonteaching & 6.1 & & 58.5 \\
Primary payer (\%) & & 46.7 & 19.9 \\
Private insurance & 49.9 & 24.7 & 14.2 \\
Medicare & 29.5 & 19.6 & 0.8 \\
Medicaid & 12.6 & 0.9 & 6.7 \\
Self-pay & 1.4 & 8.1 & \\
Other/unknown & 6.6 & & \\
\hline
\end{tabular}

Table 3. Hospital mortality, overall and by VAD BTT status for patients undergoing heart transplantation in the United States from 2003-2007.

\begin{tabular}{cccc}
\hline & Transplants & Deaths & Mortality (\%) \\
\hline All & 7859 & 487 & 6.2 \\
No BTT & 6227 & 261 & 4.2 \\
VAD Outpt & 983 & 60 & 6.1 \\
VAD Inpt & 649 & 166 & 25.6 \\
\hline
\end{tabular}

A multivariable analysis was performed using available NIS clinical data to understand whether bridging to transplantation with a VAD as either an inpatient or an outpatient was an independent risk factor for hospital mortality (Table 4). As with other previously published studies, age and underlying diagnosis of congenital heart disease were independent risk factors for hospital mortality following heart transplantation [5,7,18-23]. Outpatient BTT showed a trend toward higher hospital mortality but did not reach statistical significance. This finding also is consistent with previously published studies which have shown variable effects of bridging to transplant with implantable left ventricular assist devices, which is the likely form of circulatory support represented by this NIS population. As seen in the unadjusted data, inpatient BTT status was found to be the strongest predictor of hospital mortality, and was associated with a nine-fold increased risk of hospital mortality following heart transplantation.

Inpatient VAD support prior to transplant also substantially increased the hospital cost associated with the heart transplant admission (Table 4). The median inpa- tient cost of heart transplantation for patients who were not supported with a VAD was $\$ 99,000$ (2007 healthcare dollars). Patients who were supported as outpatients had a modestly increased cost $(\$ 17,174)$ that did not reach statistical significance. Inpatient VAD support was associated with a more than doubling of hospital cost associated with heart transplantation. Over time, the overall inpatient cost of heart transplantation declined from $\$ 115,868$ to $\$ 103,578$ (2007 healthcare dollars) (Figure 2). This improvement was driven by the decreasing cost of transplanting VAD Outpts.

The increase in hospital cost among VAD Inpts was associated with a substantially longer hospital length of stay (Table 5). The mean LOS among VAD Inpts was approximately four-fold higher than that observed for VAD Outpts and patients who were not bridged to transplant. The majority of this increased LOS was related to longer pre-transplant hospitalization among VAD Inpts. The post-transplant LOS was similar between the three groups.

\section{Discussion}

This study demonstrates an increasing utilization of VADs as bridge to transplantation in the United States from the years 2003-2007. We further analyzed the changes in the clinical setting of VAD support that may have occurred during the study period. The NIS database did allow us to identify patients who underwent their VAD implant procedure at a separate hospitalization from their heart transplant procedure and therefore were supported with a VAD as an outpatient prior to being 
Table 4. Multivariable analysis of association of demographic factors and VAD BTT status on hospital mortality and cost of transplant hospitalization.

\begin{tabular}{|c|c|c|c|c|}
\hline \multirow{2}{*}{$\begin{array}{c}\text { Response Variable } \\
\text { Variable }\end{array}$} & \multicolumn{2}{|c|}{ Hospital Mortality } & \multicolumn{2}{|c|}{ Cost (2007 healthcare dollars) } \\
\hline & Odds Ratio (95\% CI) & p value & Cost difference (95\% CI) (\$) & p value \\
\hline Age & $1.044(1.022,1.065)$ & $<0.0001$ & $-195(-546,154)$ & 0.2690 \\
\hline Female gender & $1.375(0.882,2.145)$ & 0.1603 & $9151(1030,17,272)$ & 0.0278 \\
\hline Underlying diagnosis & & 0.0009 & & 0.0005 \\
\hline Ischemia & $1.112(0.776,1.593)$ & 0.5627 & $2681(-6220,11,582)$ & 0.5496 \\
\hline Cardiomyopathy & 1.0 (Reference) & & 0.0 (Reference) & \\
\hline VAD status & & $<0.0001$ & & $<0.0001$ \\
\hline No BTT & 1.0 (Reference) & & 0.0 (Reference) & \\
\hline VAD Outpt & $1.716(0.939,3.136)$ & 0.0795 & $17,174(2654,31,694)$ & 0.0212 \\
\hline VAD Inpt & $9.904(5.959,16.462)$ & $<0.0001$ & $146,273(116,101,176,446)$ & $<0.0001$ \\
\hline
\end{tabular}

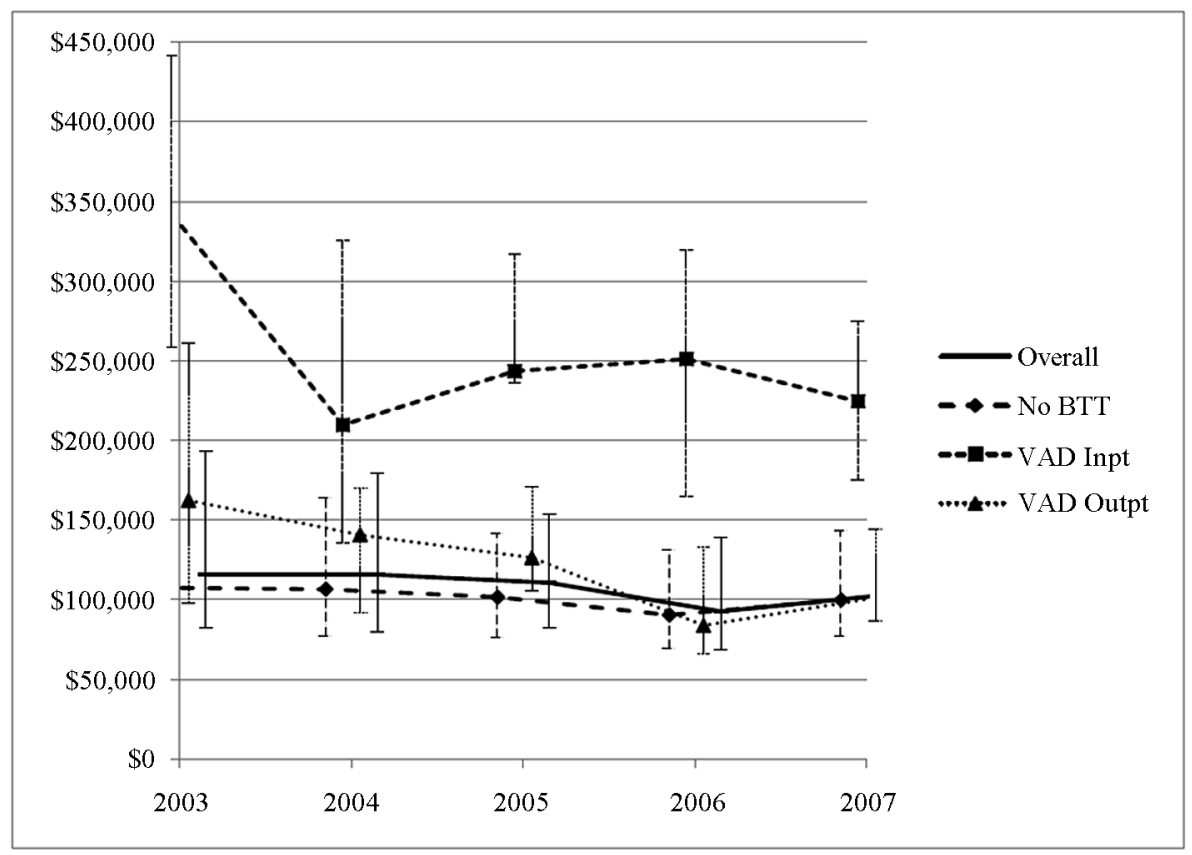

Figure 2. Median cost, overall and split by VAD status. Cost expressed in 2007 healthcare dollars.

transplanted. When segregated in this fashion, it accounted for the increase in the overall frequency of VAD utilization as a bridge to transplantation. The increase in VAD utilization was driven primarily by increases in VAD support and wait listing in an outpatient setting. The proportion of patients who underwent VAD implantation and heart transplantation while remaining as inpatients was stable throughout the study period.

This shift in treatment pathway was made possible by improvements in implantable VAD technology occurring over this time period that have made outpatient circulatory support increasingly safe $[24,25]$. The shift toward outpatient VAD support and wait listing was justified by the clinical outcomes of patients transplanted via this pathway. Patients supported as outpatients had similar hospital mortality for their heart transplant procedure as patients who were not bridged to transplant with a VAD.

Patients bridged to transplant in an inpatient setting had a markedly higher mortality in contrast. An inpatient setting for wait listing with a VAD was the strongest independent predictor of mortality identified. The poor early results of heart transplantation and the greatly increased cost of the heart transplant hospitalization associated with this pathway underscore the value to the patient and healthcare system of timely referral of the advanced heart failure patient whom are candidates for 
Table 5. Hospital length of stay (LOS), pre- and post-operative LOS by BTT status for heart transplant hospitalization. Values are median with interquartile range in parentheses.

\begin{tabular}{ccccc}
\hline & No BTT & VAD Outpt & VAD Inpt & All \\
\hline LOS, total, days & $17(11-37)$ & $19(12-36)$ & $63(36-115)$ & $20(11-42)$ \\
Pre operative LOS, days & $1(0-15)$ & $1(0-14)$ & $38(14-82)$ & $1(0-19)$ \\
Post operative LOS, days & $13(9-18)$ & $16(10-24)$ & $21(12-32)$ & $13(10-20)$ \\
\hline
\end{tabular}

implantable LVAD systems that allow a period of end organ recovery and wait listing as outpatients. It also raises the possibility that patients salvaged with external LVADs or BiVADs may have improved survival at the time of subsequent heart transplantation if they can be transitioned to an implantable LVAD that allows for outpatient recovery and wait listing. From a health system perspective, the more frequent use of the VAD Outpt versus the VAD Inpt pathway led to decreased overall hospital costs associated with heart transplantation over the study period.

This study demonstrates the feasibility of using administrative databases to study both clinical and financial outcomes of advanced heart failure patients. In the current era of heathcare reform, it is important for fields with high resource utilization to carefully consider their impact on the healthcare system. Further study is necessary to define the cost-benefit relationship of different treatments and care pathways for patients with advanced heart failure.

Unfortunately, the NIS database does not provide sufficient clinical information to better characterize the VAD Inpt group to understand what specific clinical characteristics portend hospital mortality at the time of transplantation [14]. Further study is needed to identify risk factors such as VAD device type and degree of end organ failure that may be modified to improve outcomes at the time of heart transplantation. Ideally, future studies will allow the design of algorithms that direct patient care toward pathways, such as outpatient mechanical circulatory support, that are associated with improved outcomes to both the patient and healthcare system.

\section{Acknowledgements}

This work was supported in part by Health Resources and Services Administration contract 234-2005-370011C. The content is the responsibility of the authors alone and does not necessarily reflect the views or policies of the Department of Health and Human Services, nor does mention of trade names, commercial products, or organizations imply endorsement by the US Government.

\section{REFERENCES}

[1] L. W. Miller, F. D. Pagani, S. D. Russell, R. John, A. J.
Boyle, K. D. Aaronson, et al., "Use of a Continuous-Flow Device in Patients Awaiting Heart Transplantation," The New England Journal of Medicine, Vol. 357, No. 9, 2007, pp. 885-896. doi:10.1056/NEJMoa067758

[2] D. Esmore, D. Kaye, P. Spratt, R. Larbalestier, P. Ruygrok, S. Tsui, et al., "A Prospective, Multicenter Trial of the Ventrassist Left Ventricular Assist Device for Bridge to Transplant: Safety and Efficacy," The Journal of Heart and Lung Transplantation, Vol. 27, No. 6, 2008, pp. 579588. doi:10.1016/j.healun.2008.02.012

[3] M. C. Deng, L. B. Edwards, M. I. Hertz, A. W. Rowe, B. M. Keck, R. Kormos, et al., "Mechanical Circulatory Support Device Database of the International Society for Heart and Lung Transplantation: Third Annual Report2005," The Journal of Heart and Lung Transplantation, Vol. 24, No. 9, 2005, pp. 1182-1187.

doi:10.1016/j.healun.2005.07.002

[4] O. H. Frazier, E. A. Rose, M. C. Oz, W. Dembitsky, P. McCarthy, B. Radovancevic, et al., "Multicenter Clinical Evaluation of the Heartmate Vented Electric Left Ventricular Assist System in Patients Awaiting Heart Transplantation," The Journal of Thoracic and Cardiovascular Surgery, Vol. 122, No. 6, 2001, pp. 1186-1195. doi:10.1067/mtc.2001.118274

[5] J. K. Kirklin, D. C. Naftel, L. W. Stevenson, R. L. Kormos, F. D. Pagani, M. A. Miller, et al., "INTERMACS Database for Durable Devices for Circulatory Support: First Annual Report," The Journal of Heart and Lung Transplantation, Vol. 27, No.10, 2008, pp. 1065-1072. doi:10.1016/j.healun.2008.07.021

[6] L. W. Miller, K. E. Nelson, R. R. Bostic, K. Tong, M. S. Slaughter and J. W. Long, "Hospital Costs for Left Ventricular Assist Devices for Destination Therapy: Lower Costs for Implantation in the Post-REMATCH Era," The Journal of Heart and Lung Transplantation, Vol. 25, No. 7, 2006, pp. 778-784. doi:10.1016/j.healun.2006.03.010

[7] S. E. Sandner, D. Zimpfer, P. Zrunek, A. Rajek, H. Schima, D. Dunkler, et al., "Age and Outcome after Continuous-Flow Left Ventricular Assist Device Implantation as Bridge to Transplantation," The Journal of Heart and Lung Transplantation, Vol. 28, No. 4, 2009, pp. 367-372. doi:10.1016/j.healun.2009.01.008

[8] M. C. Deng, M. Loebe, A. El-Banayosy, E. Gronda, P. G. Jansen, M. Vigano, et al., "Mechanical Circulatory Support for Advanced Heart Failure: Effect of Patient Selection on Outcome," Circulation, Vol. 103, No. 2, 2001, pp. 231-237. doi:10.1161/01.CIR.103.2.231

[9] V. Patlolla, R. D. Patten, D. Denofrio, M. A. Konstam and R. Krishnamani, "The Effect of Ventricular Assist Devices on Post-Transplant Mortality an Analysis of the 
United Network for Organ Sharing Thoracic Registry," Journal of the American College of Cardiology, Vol. 53, No. 3, 2009, pp. 264-271. doi:10.1016/j.jacc.2008.08.070

[10] J. S. Gammie, L. B. Edwards, B. P. Griffith, R. N. Griffith and L. Tsao, "Optimal Timing of Cardiac Transplantation after Ventricular Assist Device Implantation," The Journal of Thoracic and Cardiovascular Surgery, Vol. 127, No. 6, 2004, pp. 1789-1799. doi:10.1016/i.jtcvs.2003.12.018

[11] R. John, F. D. Pagani, Y. Naka, A. Boyle, J. V. Conte, S. D. Russell, et al., "Post-Cardiac Transplant Survival after Support with a Continuous-Flow Left Ventricular Assist Device: Impact of Duration of Left Ventricular Assist Device Support and Other Variables," The Journal of Thoracic and Cardiovascular Surgery, Vol. 140, No. 1, 2010, pp. 174-181. doi:10.1016/j.jtcvs.2010.03.037

[12] "Introduction to the HCUP Nationwide Inpatient Sample (NIS) 2007," Healthcare Cost and Utilization Project (HCUP), Agency for Healthcare Research and Quality, US Department of Health and Human Services, 2009.

[13] "2009 Annual Report of the US Organ Procurement and Transplantation Network and the Scientific Registry of Transplant Recipients: Transplant Data 1997-2008," Department of Health and Human Services, Health Resources and Services Administration, Healthcare Systems Bureau, Division of Transplantation, Rockville, United Network for Organ Sharing, Richmond, University Renal Research and Education Association, Ann Arbor.

[14] S. M. Martinelli, U. D. Patel, B. G. Phillips-Bute, C. A. Milano, L. E. Archer, M. Stafford-Smith, et al., "Trends in Cardiac Surgery-Associated Acute Renal Failure in the United States: A Disproportionate Increase after Heart Transplantation," Renal Failure, Vol. 31, No. 8, 2009, pp. 633-640. doi:10.3109/08860220903100689

[15] D. Chu, F. G. Bakaeen, T. K. Dao, S. A. LeMaire, J. S. Coselli and J. Huh, "On-Pump versus Off-Pump Coronary Artery Bypass Grafting in a Cohort of 63,000 Patients," The Annals of Thoracic Surgery, Vol. 87, No. 6, 2009, pp. 1820-1826. doi:10.1016/j.athoracsur.2009.03.052

[16] H. Luckraz, M. Goddard, S. C. Charman, J. Wallwork, J. Parameshwar and S. R. Large, "Early Mortality after Cardiac Transplantation: Should We Do Better?" The Journal of Heart and Lung Transplantation, Vol. 24, No. 4, 2005, pp. 401-405. doi:10.1016/j.healun.2004.02.013

[17] P. Demers, S. Moffatt, P. E. Oyer, S. A. Hunt, B. A. Reitz and R. C. Robbins, "Long-Term Results of Heart Transplantation in Patients Older than 60 Years," The Journal of Thoracic and Cardiovascular Surgery, Vol. 126, No. 1,
2003, pp. 224-231. doi:10.1016/S0022-5223(03)00055-2

[18] V. K. Topkara, N. C. Dang, T. P. Martens, F. H. Cheema, J. F. Liu, M. Argenziano, et al., "Bridging to Transplantation with Left Ventricular Assist Devices: Outcomes in Patients Aged 60 Years and Older," The Journal of Thoracic and Cardiovascular Surgery, Vol. 130, No. 3, 2005, pp. 881-882. doi:10.1016/j.jtcvs.2005.03.009

[19] P. L. Digiorgi, M. S. Reel, B. Thornton, E. Burton, Y. Naka and M. C. Oz, "Heart Transplant and Left Ventricular Assist Device Costs," The Journal of Heart and Lung Transplantation, Vol. 24, No. 2, 2005, pp. 200-204. doi:10.1016/j.healun.2003.11.397

[20] A. F. Hernandez, A. M. Shea, C. A. Milano, J. G. Rogers, B. G. Hammill, C. M. O'Connor, et al., "Long-Term Outcomes and Costs of Ventricular Assist Devices among Medicare Beneficiaries," JAMA: The Journal of the American Medical Association, Vol. 300, No. 20, 2008, pp. 2398-2406. doi:10.1001/jama.2008.716

[21] A. J. Moskowitz, E. A. Rose and A. C. Gelijns, "The Cost of Long-Term LVAD Implantation," The Annals of Thoracic Surgery, Vol. 71, Supplement 3, 2001, pp. S195S198. doi:10.1016/S0003-4975(00)02621-7

[22] S. Osaki, N. M. Edwards, M. R. Johnson, M. Velez, A. Munoz, L. Lozonschi, et al., "Improved Survival after Heart Transplantation in Patients with Bridge to Transplant in the Recent Era: A 17-Year Single-Center Experience," The Journal of Heart and Lung Transplantation, Vol. 28, No. 6, 2009, pp. 591-597. doi:10.1016/j.healun.2009.03.008

[23] J. A. Morgan, R. John, V. Rao, A. D. Weinberg, B. J. Lee, P. A. Mazzeo, et al., "Bridging to Transplant with the HeartMate Left Ventricular Assist Device: The Columbia Presbyterian 12-Year Experience," The Journal of Thoracic and Cardiovascular Surgery, Vol. 127, No. 5, 2004, pp. 1309-1316. doi:10.1016/j.jtcvs.2003.07.035

[24] J. MacIver, H. J. Ross, D. H. Delgado, R. J. Cusimano, T. M. Yau, M. Rodger, et al., "Community Support of Patients with a Left Ventricular Assist Device: The Toronto General Hospital Experience," The Canadian Journal of Cardiology, Vol. 25, No. 11, 2009, pp. e377-e381. doi:10.1016/S0828-282X(09)70164-3

[25] D. L. Morales, K. A. Catanese, D. N. Helman, M. R. Williams, A. Weinberg, D. J. Goldstein, et al., "Six-Year Experience of Caring for Forty-Four Patients with a Left Ventricular Assist Device at Home: Safe, Economical, Necessary," The Journal of Thoracic and Cardiovascular Surgery, Vol. 119, No. 2, 2000, pp. 251-259. doi:10.1016/S0022-5223(00)70180-2 